Proceedings of the 8th Polish Symposium of Physics in Economy and Social Sciences FENS, Rzeszów, November 4-6, 2015

\title{
On the Foster-Hart Measure of Riskiness under Cumulative Prospect Theory
}

\author{
J. ChudZiak ${ }^{a * *}$ AND M. HALICKI ${ }^{b}$ \\ ${ }^{a}$ Faculty of Mathematics and Natural Sciences, University of Rzeszów, Prof. St. Pigonia 1, 35-310 Rzeszów, Poland \\ ${ }^{b}$ Department of Regional Politics and Food Economy, University of Rzeszów, \\ M. Ćwiklińskiej 2, 35-601 Rzeszów, Poland
}

\begin{abstract}
We prove the existence of the Foster-Hart measure of riskiness under the cumulative prospect theory and we study some of its basic properties.
\end{abstract}

DOI: 10.12693/APhysPolA.129.950

PACS/topics: 89.65.Gh

\section{Introduction}

Recently one can observe an increasing interest in various approaches to risk measures. One of them consists on listing a desirable properties (axioms) that such a risks measure should satisfy and then on deriving their functional representations. The axiomatic approach has been propagated by Artzner et al. [1], who introduced the notion of a coherent risk measure and by Föllmer and Schied [2], who have investigated convex risk measures. Roughly speaking, a risk measure is a functional, assigning a real number to the random variable, describing the risk related to some financial position. In Ref. [3] a new measure of riskiness has been introduced. In order to define the measure, we shall assume that $\Delta$ is a family of all finitely-valued random variables $X$, defined on a given probability space, such that $P(X<0)>0$ and $E(X)>0$. In what follows, by $\left(x_{1}, p_{1} ; x_{2}, p_{2} ; \ldots ; x_{n}, p_{n}\right)$ we denote a random variable taking the values $x_{1}, \ldots, x_{n}$ with probabilities $p_{1}, \ldots, p_{n}$, respectively. Furthermore, we set

$$
L(X):=-\min X \quad \text { for } \quad X \in \Delta,
$$

that is, $L(X)$ is a maximal loss related to $X$. In Ref. [3] it has been proved that for every $X \in \Delta$ there exists a unique real number $R(X)>L(X)$ such that

$$
E\left(\ln \left(1+\frac{X}{R(X)}\right)\right)=0 .
$$

A functional $R: \Delta \rightarrow \mathbb{R}$ defined in this way is called a Foster-Hart measure of riskiness. Foster and Hart [3] have proved the following result. Suppose that $W_{1}>0$ is a decision maker's initial wealth. At every period $n \in \mathbb{N}$, the decision maker with a current wealth $W_{n}$ is offered a gamble $X_{n} \in \Delta$. If he accepts $X_{n}$, then his wealth in the next period is $W_{n+1}=W_{n}+X_{n}$; otherwise it is $W_{n+1}=W_{n}$. If, for every $n \in \mathbb{N}$, the decision maker accepts a gamble if and only if $W_{n} \geq R\left(X_{n}\right)$, then he avoids bankruptcy almost surely. For the general (not

\footnotetext{
*corresponding author; e-mail: chudziak@ur.rzeszow.pl
}

necessarily finitely-valued) random variables, the FosterHart measure has been considered by Riedel and Hellmann [4]. A dynamic extension of the measure has been investigated in [5].

Let us illustrate this concept with the following simple example. Suppose that, at some period $n \in \mathbb{N}$, a decision maker has to decide to buy or not a risky asset which offers, with equal probabilities, the payoffs -90 and 108. Such an asset is represented by a random variable $X_{n}=(-90,0.5 ; 108,0.5)$. Moreover, as $P\left(X_{n}<0\right)=P\left(X_{n}=-90\right)=0.5>0$ and $E\left(X_{n}\right)=$ $0.5 \times(-90)+0.5 \times 108=9>0$, we have $X_{n} \in \Delta$. According to (2), the Foster-Hart measure of $X_{n}$ is a unique solution $R\left(X_{n}\right)>L\left(X_{n}\right)=90$ of the equation

$$
0.5 \ln \left(1-\frac{90}{R\left(X_{n}\right)}\right)+0.5 \ln \left(1+\frac{108}{R\left(X_{n}\right)}\right)=0 .
$$

A standard calculation shows that $R\left(X_{n}\right)=540$. So, if the decision maker's wealth at the considered period is at least 540, he should buy the asset; otherwise, he should reject it.

In Ref. [3] several properties of the functional $R$ has been proved. In particular, it has been shown there that:

- $R$ is positively homogeneous, i.e. $R(\lambda X)=\lambda R(X)$ for $X \in \Delta, \lambda \in(0, \infty)$,

- $R$ is subadditive, i.e. $R(X+Y) \leq R(X)+R(Y)$ for $X, Y \in \Delta$,

- $R$ has the dilution property $R(\alpha \star X)=R(X)$ for $X \in \Delta, \alpha \in[0,1]$, where, for every $\alpha \in[0,1]$ and $X=$ $\left(x_{1}, p_{1} ; x_{2}, p_{2} ; \ldots ; x_{n}, p_{n}\right) \in \Delta$, $\alpha \star X:=\left(x_{1}, \alpha p_{1} ; x_{2}, \alpha p_{2} ; \ldots ; x_{n}, \alpha p_{n} ; 0,1-\alpha\right)$.

The aim of this note is to prove the existence and to study the above properties of the Foster-Hart measure of riskiness under the cumulative prospect theory. The cumulative prospect theory is a model of decision making under risk. The theory has been created by Tversky and Kahneman [6] on a base of several experiments, showing that, making decisions under risk, people set a reference point and consider the lower outcomes as losses and 
larger as gains. Furthermore, people distort probabilities. In general, the probabilities of gains and losses are distorted in different ways. According to their observations, Tversky and Kahneman introduced the generalized Choquet integral and applied it to describe the mathematical foundations of the cumulative prospect theory.

Let us recall that the Choquet integral with respect to a probability distortion function $g$, that is a non-decreasing function $g:[0,1] \rightarrow[0,1]$ with $g(0)=0$ and $g(1)=1$, is defined in the following way:

$$
\begin{aligned}
& E_{g}(X)=\int_{-\infty}^{0}(g(P(X>t))-1) \mathrm{d} t \\
& \quad+\int_{0}^{\infty} g(P(X>t)) \mathrm{d} t .
\end{aligned}
$$

The generalized Choquet integral, related to the distortion functions $g$ (for gains) and $h$ (for losses), is defined as follows:

$$
E_{g h}(X)=E_{g}(\max \{X, 0\})-E_{h}(\max \{-X, 0\}) .
$$

\section{Results}

In this section we assume that $g$ and $h$ are probability distortion functions for gains and losses, respectively and $\Delta$ is a family of all finitely-valued random variables $X$, defined on a given probability space, such that $P(X<0)>0$ and $E_{g h}(X)>0$. We begin with the result concerning the existence of the Foster-Hart measure of riskiness under the cumulative prospect theory.

Theorem 2.1: For every $X \in \Delta$ there exists a unique real number $R(X)>L(X)$ such that

$$
E_{g h}(\ln (1+X / R(X)))=0 .
$$

Moreover, for every $t \in\left(0, \frac{1}{L(X)}\right)$, we have

$$
E_{g h}(\ln (1+t X))>0 \quad \text { if and only if } t<\frac{1}{R(X)} .
$$

In the next results we present the properties of the Foster-Hart measure under the cumulative prospect theory.

Proposition 2.2: The Foster-Hart measure of riskiness under the cumulative prospect theory is positively homogeneous.

Proposition 2.3: Assume that $g$ is a convex function and $h(p)=1-g(1-p)$ for $p \in[0,1]$. Then the FosterHart measure of riskiness under the cumulative prospect theory is subadditive.

Remark 2.4: From Propositions 2.2 and 2.3 it follows that if $g$ is a convex function and $h(p)=1-g(1-p)$ for $p \in[0,1]$, then the Foster-Hart measure of riskiness under the cumulative prospect theory is convex.

Theorem 2.5: If $g(p)>0$ for $p \in(0,1]$ and the Foster-Hart measure of riskiness under the cumulative prospect theory has the dilution property, then there exists an $r \in(0, \infty)$ such that

$$
g(p)=h(p)=p^{r} \quad \text { for } p \in[0,1] .
$$

Conversely, if (7) holds with some $r \in(0, \infty)$, then the Foster-Hart measure of riskiness under the cumulative prospect theory has the dilution property.
Remark 2.6: As we have already mentioned, Foster and Hart [3] have proved that if at every period $n \in \mathbb{N}$ the decision maker, with a current wealth $W_{n}$, accepts a game $X_{n}$ if and only if $W_{n} \geq R\left(X_{n}\right)$, then he avoids bankruptcy almost surely. It is an open problem, if the same result is true in the case of the Foster-Hart measure of riskiness under the cumulative prospect theory.

\section{Conclusion}

In Ref. [3] a new operational measure of riskiness has been introduced. It has been proved there that the measure has several interesting properties. In this paper we introduce the analogous measure of riskiness under the cumulative prospect theory. First, we prove the existence of such a measure. Then, we show that under the cumulative prospect theory, the Foster-Hart measure is positively homogeneous. Moreover, it is subadditive provided the probability distortion functions for gains and loses satisfy some additional assumptions. Finally, the measure has the dilution property if and only if the probability distortion functions for gains and losses are equal and both are the power functions.

\section{Proofs}

Every $X \in \Delta$ can be expressed in the form $X=$ $\left(x_{1}, p_{1} ; x_{2}, p_{2} ; \ldots ; x_{n}, p_{n}\right)$ with some $n \in \mathbb{N}$ and $x_{1}<$ $x_{2}<\ldots<x_{n}$. Then the condition $P(X<0)>0$ implies that $x_{1}<0$ and so, in view of (1), we get

$$
L(X)=-x_{1} .
$$

Furthermore, as the Choquet integral is monotone and $E_{g h}(c)=c$ for $c \in \mathbb{R}$, from the assumption $E_{g h}(X)>0$ it follows that $x_{n}>0$. Thus,

$$
k:=\min \left\{i \in\{2, \ldots, n\}: x_{i}>0\right\}
$$

is well defined. Moreover, we have

$$
\begin{aligned}
& \max \{X, 0\}=\left(0, \sum_{s=1}^{k-1} p_{s} ; x_{k}, p_{k} ; x_{k+1}, p_{k+1} ; \ldots ; x_{n}, p_{n}\right), \\
& \max \{-X, 0\}=\left(0, \sum_{s=k-1}^{n} p_{s} ;-x_{k-2}, p_{k-2} ; \ldots ;-x_{1}, p_{1}\right)
\end{aligned}
$$$$
\text { whenever } x_{k-1}=0
$$

and

$$
\max \{-X, 0\}=\left(0, \sum_{s=k}^{n} p_{s} ;-x_{k-1}, p_{k-1} ; \ldots ;-x_{1}, p_{1}\right)
$$

whenever $x_{k-1}<0$.

Hence, adopting the convention $\sum_{i=l}^{k}=0$ whenever $l>k$, in view of $(3)$, we obtain

$$
\begin{gathered}
E_{g}(\max \{X, 0\})=\int_{0}^{x_{k}} g\left(\sum_{s=k}^{n} p_{s}\right) \mathrm{d} t \\
+\sum_{i=k}^{n-1} \int_{x_{i}}^{x_{i+1}} g\left(\sum_{s=i+1}^{n} p_{s}\right) \mathrm{d} t=
\end{gathered}
$$


$g\left(\sum_{s=k}^{n} p_{s}\right) x_{k}+\sum_{i=k}^{n-1} g\left(\sum_{s=i+1}^{n} p_{s}\right)\left(x_{i+1}-x_{i}\right)$.

Similarly, we get

$$
\begin{gathered}
E_{h}(\max \{-X, 0\})=h\left(\sum_{s=1}^{k-1} p_{s}\right)\left(-x_{k-1}\right) \\
+\sum_{i=2}^{k-1} h\left(\sum_{s=1}^{i-1} p_{s}\right)\left(-x_{i-1}+x_{i}\right) .
\end{gathered}
$$

Therefore, according to (4), we have

$$
\begin{aligned}
& E_{g h}(X)=g\left(\sum_{s=k}^{n} p_{s}\right) x_{k}+h\left(\sum_{s=1}^{k-1} p_{s}\right) x_{k-1} \\
& +\sum_{i=k}^{n-1} g\left(\sum_{s=i+1}^{n} p_{s}\right)\left(x_{i+1}-x_{i}\right) \\
& +\sum_{i=2}^{k-1} h\left(\sum_{s=1}^{i-1} p_{s}\right)\left(x_{i-1}-x_{i}\right) .
\end{aligned}
$$

The following lemma plays a crucial role in the proof of Theorem 2.1.

Lemma 4.1: Assume that $X=$ $\left(x_{1}, p_{1} ; x_{2}, p_{2} ; \ldots ; x_{n}, p_{n}\right) \in \Delta$, where $n \in \mathbb{N}$ and $x_{1}<x_{2}<\ldots<x_{n}$. Let a function $\phi:\left(-\frac{1}{x_{n}},-\frac{1}{x_{1}}\right) \rightarrow \mathbb{R}$ be given by

$$
\phi(t)=E_{g h}(\ln (1+t X)) \quad \text { for } t \in\left(-\frac{1}{x_{n}},-\frac{1}{x_{1}}\right) .
$$

Then there exists a unique $t_{0} \in\left(0,-\frac{1}{x_{1}}\right)$ such that $\phi\left(t_{0}\right)=0$. Furthermore, for every $t \in\left(0,-\frac{1}{x_{1}}\right)$, it holds

$$
\phi(t)>0 \text { if and only if } t \in\left(0, t_{0}\right) .
$$

Proof. Let $k$ be defined by (9). Since, for every $t \in$ $\left(-\frac{1}{x_{n}},-\frac{1}{x_{1}}\right)$, we have

$$
\begin{aligned}
& \ln (1+t X)=\left(\ln \left(1+t x_{1}\right), p_{1} ; \ln \left(1+t x_{2}\right), p_{2} ; \ldots ;\right. \\
& \left.\quad \ln \left(1+t x_{n}\right), p_{n}\right),
\end{aligned}
$$

making use of (10) and (11), we obtain

$$
\begin{aligned}
\phi(t) & =g\left(\sum_{s=k}^{n} p_{s}\right) \ln \left(1+t x_{k}\right) \\
+ & \sum_{i=k}^{n-1} g\left(\sum_{s=i+1}^{n} p_{s}\right) \ln \left(\frac{1+t x_{i+1}}{1+t x_{i}}\right) \\
+ & h\left(\sum_{s=1}^{k-1} p_{s}\right) \ln \left(1+t x_{k-1}\right) \\
+ & \sum_{i=2}^{k-1} h\left(\sum_{s=1}^{i-1} p_{s}\right) \ln \left(\frac{1+t x_{i-1}}{1+t x_{i}}\right) .
\end{aligned}
$$

Hence, for every $t \in\left(-\frac{1}{x_{n}},-\frac{1}{x_{1}}\right)$, we get

$$
\begin{aligned}
\phi^{\prime}(t) & =\frac{g\left(\sum_{s=k}^{n} p_{s}\right) x_{k}}{1+t x_{k}} \\
+ & \sum_{i=k}^{n-1} g\left(\sum_{s=i+1}^{n} p_{s}\right) \\
\times & \left(\frac{x_{i+1}}{1+t x_{i+1}}-\frac{x_{i}}{1+t x_{i}}\right)+\frac{h\left(\sum_{s=1}^{k-1} p_{s}\right) x_{k-1}}{1+t x_{k-1}} \\
+ & \sum_{i=2}^{k-1} h\left(\sum_{s=1}^{i-1} p_{s}\right)\left(\frac{x_{i-1}}{1+t x_{i-1}}-\frac{x_{i}}{1+t x_{i}}\right)
\end{aligned}
$$

and

$$
\begin{aligned}
& \phi^{\prime \prime}(t)=-\frac{g\left(\sum_{s=k}^{n} p_{s}\right) x_{k}^{2}}{\left(1+t x_{k}\right)^{2}}-\sum_{i=k}^{n-1} g\left(\sum_{s=i+1}^{n} p_{s}\right) \\
& \times\left(\frac{x_{i+1}^{2}}{\left(1+t x_{i+1}\right)^{2}}-\frac{x_{i}^{2}}{\left(1+t x_{i}\right)^{2}}\right)-\frac{h\left(\sum_{s=1}^{k-1} p_{s}\right) x_{k-1}^{2}}{\left(1+t x_{k-1}\right)^{2}} \\
& -\sum_{i=2}^{k-1} h\left(\sum_{s=1}^{i-1} p_{s}\right)\left(\frac{x_{i-1}^{2}}{\left(1+t x_{i-1}\right)^{2}}-\frac{x_{i}^{2}}{\left(1+t x_{i}\right)^{2}}\right) .
\end{aligned}
$$

Furthermore, the straightforward calculations show that

$$
\begin{aligned}
& \frac{x_{i+1}^{2}}{\left(1+t x_{i+1}\right)^{2}}-\frac{x_{i}^{2}}{\left(1+t x_{i}\right)^{2}}>0 \\
& \quad \text { for } i \in\{k, \ldots, n-1\}, t \in\left(0,-\frac{1}{x_{1}}\right)
\end{aligned}
$$

and

$$
\begin{aligned}
& \frac{x_{i-1}^{2}}{\left(1+t x_{i-1}\right)^{2}}-\frac{x_{i}^{2}}{\left(1+t x_{i}\right)^{2}}>0 \\
& \text { for } i \in\{2, \ldots, k-1\}, t \in\left(0,-\frac{1}{x_{1}}\right) .
\end{aligned}
$$

Thus $\phi^{\prime \prime}(t)<0$ for $t \in\left(0,-\frac{1}{x_{1}}\right)$, that is $\phi$ is concave on the interval $\left(0,-\frac{1}{x_{1}}\right)$. Moreover, in view of $(10),(13)$ and (14), we obtain that $\phi^{\prime}(0)=E_{g h}(X)>0$ and $\phi(0)=0$. Hence, a set $T:=\{\varepsilon>0: \phi(t)>0$ for $t \in(0, \varepsilon)\}$, is nonempty and bounded above. Let $t_{0}:=\sup T$. Then, obviously, $\phi(t)>0$ for $t \in\left(0, t_{0}\right)$. Furthermore, by (13), we get $\lim _{t \rightarrow-\frac{1}{x_{1}}}-\phi(t)=-\infty$. Hence $t_{0}<-\frac{1}{x_{1}}$ and so, as $\phi$ is continuous, we conclude that $\phi\left(t_{0}\right)=0$. Therefore, in order to complete the proof, it is enough to show that $\phi(t)<0$ for $t \in\left(t_{0},-\frac{1}{x_{1}}\right)$. To this end, fix a $t \in\left(t_{0},-\frac{1}{x_{1}}\right)$ and put $\alpha:=\frac{t_{0}}{2 t-t_{0}}$. Then $\alpha \in(0,1)$ and $t_{0}=(1-\alpha) \frac{t_{0}}{2}+\alpha t$. Since $\phi$ is concave, this implies that $0=\phi\left(t_{0}\right) \geq(1-\alpha) \phi\left(\frac{t_{0}}{2}\right)+\alpha \phi(t)$ and so $\phi(t) \leq\left(1-\frac{1}{\alpha}\right) \phi\left(\frac{t_{0}}{2}\right)<0$.

\subsection{Proof of Theorem 2.1}

Proof. Taking into account (8), from Lemma 4.1 we derive that for every $X \in \Delta$ there exists a unique $t_{0} \in$ 
$\left(0, \frac{1}{L(X)}\right)$ such that $\phi\left(t_{0}\right)=0$, where $\phi:\left(-\frac{1}{x_{n}},-\frac{1}{x_{1}}\right) \rightarrow$ $\mathbb{R}$ is given by (11). So, taking $R(X):=\frac{1}{t_{0}}$, we get (5). A uniqueness of $R(X)$ follows from a uniqueness of $t_{0}$. Furthermore, from (12) we derive (6).

\subsection{Proof of Proposition 2.2}

Proof. Let $X \in \Delta$ and $\lambda \in(0, \infty)$. Applying Theorem 2.1 and taking into account (1), we get $\lambda R(X)>\lambda L(X)=$ $L(\lambda X)$ and

$$
E_{g h}\left(\ln \left(1+\frac{\lambda X}{\lambda R(X)}\right)\right)=E_{g h}\left(\ln \left(1+\frac{X}{R(X)}\right)\right)=0 .
$$

On the other hand, according to Theorem 2.1, a unique real number with these two properties, is $R(\lambda X)$. Hence $R(\lambda X)=\lambda R(X)$.

\subsection{Proof of Proposition 2.3}

Proof. Since $h(p)=1-g(1-p)$ for $p \in[0,1]$, it is not difficult to check that $E_{g h}=E_{g}$. Furthermore, as $g$ is convex, taking into account Example 1 on p. $16-17$ and Corollary 6.4 on p. 78 in Ref. [7], we conclude that $E_{g}$ is superadditive. Fix $X, Y \in \Delta$ and set $t:=\frac{R(X)}{R(X)+R(Y)}$. Then, obviously, $t \in(0,1)$ and

$$
\frac{X+Y}{R(X)+R(Y)}=t \frac{X}{R(X)}+(1-t) \frac{Y}{R(Y)} .
$$

Hence, as the mapping $(0, \infty) \ni x \rightarrow \ln (1+x)$ is concave and $E_{g h}=E_{g}$, making use of the superadditivity, monotonicity and positive homogeneity of $E_{g}$ (cf. Proposition 5.1 on p. 64 in Ref. [7]), in view of (5), we get

$$
\begin{aligned}
& E_{g h}\left(\ln \left(1+\frac{X+Y}{R(X)+R(Y)}\right)\right)= \\
& E_{g}\left(\ln \left(1+t \frac{X}{R(X)}+(1-t) \frac{Y}{R(Y)}\right)\right) \geq \\
& \quad E_{g}\left(t \ln \left(1+\frac{X}{R(X)}\right)+(1-t) \ln \left(1+\frac{Y}{R(Y)}\right)\right) \geq \\
& E_{g}\left(t \ln \left(1+\frac{X}{R(X)}\right)\right) \\
& +E_{g}\left((1-t) \ln \left(1+\frac{Y}{R(Y)}\right)\right)= \\
& t E_{g}\left(\ln \left(1+\frac{X}{R(X)}\right)\right)+(1-t) \\
& \quad \times E_{g}\left(\ln \left(1+\frac{Y}{R(Y)}\right)\right)=t E_{g h}\left(\ln \left(1+\frac{X}{R(X)}\right)\right) \\
& +(1-t) E_{g h}\left(\ln \left(1+\frac{Y}{R(Y)}\right)\right)=0 .
\end{aligned}
$$

Thus, making use of (6), we conclude that $\frac{1}{R(X)+R(Y)} \leq$ $\frac{1}{R(X+Y)}$, which implies that $R(X+Y) \leq R(X)+R(Y)$. Therefore, $R$ is subadditive.

\subsection{Proof of Theorem 2.5}

Proof. First, assume that (7) holds with some $r \in(0, \infty)$. Then, applying (5) and (10), after a standard computation, for every $X \in \Delta$ and $\alpha \in[0,1]$, we obtain

$$
\begin{aligned}
& E_{g h}\left(\ln \left(1+\frac{\alpha \star X}{R(X)}\right)\right)= \\
& \alpha^{r} E_{g h}\left(\ln \left(1+\frac{X}{R(X)}\right)\right)=0 .
\end{aligned}
$$

Moreover, in view of (1), we get $L(X)=L(\alpha \star X)$, so $R(X)>L(X)=L(\alpha \star X)$. According to Theorem 2.1, a unique real number with these properties is $R(\alpha \star X)$. Hence $R(\alpha \star X)=R(X)$, which means that $R$ has the dilution property.

Now, assume that $g(p)>0$ for $p \in(0,1]$ and $R$ has the dilution property. Fix $\alpha, p \in(0,1), x \in(-\infty, 0)$ and $y \in(0, \infty)$ such that

$$
y>-\frac{h(1-p)}{g(p)} x \text {. }
$$

Consider a random variable $X=(x, 1-p ; y, p)$. Note that $P(X<0)=P(X=x)=1-p>0$ and, in view of (10) and (15), we get $E_{g h}(X)=h(1-p) x+g(p) y>0$. Thus $X \in \Delta$. Furthermore, we have $\alpha \star X=(x, \alpha(1-$ $p) ; 0,1-\alpha ; y, \alpha p)$. Hence, making use of (10), we obtain

$$
\begin{aligned}
& E_{g h}\left(\ln \left(1+\frac{X}{R(X)}\right)\right)=h(1-p) \ln \left(1+\frac{x}{R(X)}\right) \\
& \quad+g(p) \ln \left(1+\frac{y}{R(X)}\right)
\end{aligned}
$$

and

$$
\begin{aligned}
& E_{g h}\left(\ln \left(1+\frac{\alpha \star X}{R(\alpha \star X)}\right)\right)=h(\alpha(1-p)) \\
& \quad \times \ln \left(1+\frac{x}{R(\alpha \star X)}\right)+g(\alpha p) \ln \left(1+\frac{y}{R(\alpha \star X)}\right) .
\end{aligned}
$$

Therefore, as $R$ has the dilution property, in view of (5), we obtain

$$
h(1-p) \ln \left(1+\frac{x}{R(X)}\right)+g(p) \ln \left(1+\frac{y}{R(X)}\right)=0
$$

and

$$
\begin{aligned}
& h(\alpha(1-p)) \ln \left(1+\frac{x}{R(X)}\right) \\
& \quad+g(\alpha p) \ln \left(1+\frac{y}{R(X)}\right)=0 .
\end{aligned}
$$

Determining $\ln \left(1+\frac{y}{R(X)}\right)$ from the first equality, substituting it into the second one and using the fact that $x \neq 0$, we get

$$
h(\alpha(1-p))-\frac{g(\alpha p)}{g(p)} h(1-p)=0 .
$$

In this way we have proved that

$$
\frac{h(\alpha(1-p))}{g(\alpha p)}=\frac{h(1-p)}{g(p)} \quad \text { for } \quad \alpha, p \in(0,1) .
$$


Let $\phi:(0,1) \rightarrow \mathbb{R}$ be given by

$$
\phi(p)=\frac{h(1-p)}{g(p)} \quad \text { for } \quad p \in(0,1) .
$$

Since $\frac{z}{p} \in(0,1)$ for $z, p \in(0,1)$ with $z<p$, applying

(16) with $\alpha:=\frac{z}{p}$, in view of (17), we obtain

$$
h\left(z\left(\frac{1}{p}-1\right)\right)=\phi(p) g(z)
$$

$$
\text { for } z, p \in(0,1), z<p \text {. }
$$

Moreover, $\frac{1}{1+u} \in(0,1)$ for $u \in(0, \infty)$, so setting in (18) $p=\frac{1}{1+u}$, we get

$$
\begin{aligned}
& h(u z)=\phi\left(\frac{1}{1+u}\right) g(z) \\
& \quad \text { for } u \in(0, \infty), 0<z<\frac{1}{1+u} .
\end{aligned}
$$

Hence, taking $D:=\left\{(u, z) \in(0, \infty) \times(0,1): 0<z<\frac{1}{1+u}\right\}$ and $\psi:(0, \infty) \rightarrow \mathbb{R}$, given by $\psi(u)=\phi\left(\frac{1}{1+u}\right)$ for $u \in(0, \infty)$, we obtain

$$
h(u z)=\psi(u) g(z) \quad \text { for } \quad(u, z) \in D .
$$

Note that

$$
\begin{aligned}
& \{u \in(0, \infty):(u, z) \in D \quad \text { for some } z \in(0,1)\}=(0, \infty), \\
& \{z \in(0,1):(u, z) \in D \quad \text { for some } u \in(0, \infty)\}=(0,1)
\end{aligned}
$$

and $\{u z:(u, z) \in D\}=(0,1)$. Thus, as $g$ and $h$ are continuous on $[0,1]$ and $g(1)=h(1)=1$, applying Corollary 2 in Sobek [8] and Theorem 13.3.6 in Kuczma [9], we obtain that there exists an $r \in(0, \infty)$ such that Eq. (7) holds.

\section{References}

[1] P. Artzner, F. Delbaen, J.-M. Eber, D. Heath, Math. Finance 9, 203 (1999).

[2] H. Föllmer, A. Schied, Finance Stoch. 6, 429 (2002).

[3] D.P. Foster, S. Hart, J. Politic. Econ. 117, 785 (2009).

[4] F. Riedel, T. Hellmann, Theor. Econom. 10, 1 (2015).

[5] T. Hellmann, F. Riedel, J. Math. Econ. 59, 66 (2015).

[6] A. Tversky, D. Kahneman, J. Risk Uncertainty 5, 297 (1992).

[7] D. Denneberg, Lectures on Non-Additive Measure and Integral, Kluwer, Boston 1994.

[8] B. Sobek, Demonstratio Math. 43, 81 (2010).

[9] M. Kuczma, An Introduction to the Theory of Functional Equations and Inequalities, Birkhäuser, Berlin 2009 . 